\title{
High frequency isolation dual-active-bridge converter with optimal control scheme based on solid-state transformer
}

\author{
Liang Wang ${ }^{1, a}$, Zhiyun $\mathrm{Bao}^{2}$ and Yi Wang ${ }^{1}$ \\ ${ }^{1}$ Power Electronic \& Motion Control Research Center, Shenzhen Graduate School of Harbin Institute of Technology, \\ Shenzhen 518055, China \\ ${ }^{2}$ Shenzhen Polytechnic, Shenzhen 518055, China
}

\begin{abstract}
This paper presents the design of high-frequency transformer isolation bidirectional DC/DC converter based on solid-state transformer (SST). A phase-shift dual-active-bridge (DAB) converter is employed to achieve high-frequency galvanic isolation, bidirectional power flow, and zero voltage switching of all switching devices, which leads to low switching losses with high frequency operation. Moreover, a master-slave control (MSC) strategy is designed for the DAB stage of SST system to reduce load on the controller and simplify modulation algorithm. The master controller executes all control and modulation calculations, and the slave controllers manage only device switching and protection. As the core circuit of SST system, DAB converter has wide applications such as battery energy storage device and hybrid electrical vehicles power system.
\end{abstract}

Keywords: solid-state transformer; dual-active-bridge; master-slave control; simplify modulation algorithm.

\section{Introduction}

The proliferation of distributed generation and renewable energy resources has motivated the researchers to investigate the feasibility of new microgrid operation mode Future Renewable Electric Energy Delivery and Management (FREEDM). The FREEDM system is a new medium-voltage microgrid composed of several solid-state transformers (SST), high-bandwidth digital communication, and distributed control. The future intelligent electric energy distribution and management systems are expected to integrate highly distributed and scalable renewable generationg sources. As the fundamental component of innovative smart microgrid system, SST is intended to replace the conventional bulky, heavy, and noisy low frequency (LF) transformers and performs the power flow control [1-2]. SST is a new power electronics system, can provide many additional advantages such as intelligent energy management, good power quality, low volum, reactive power compensation and high power density [3].

Fig. 1 shows the proposed SST based on the dual active bridge (DAB) converter and cascaded Hbridge multilevel inverter (CHMI). The SST includes a DAB DC/DC stage to step up the 400Vdc link to the high voltage dc link, a cascade H-Bridge multilevel DC/AC inverter stage to provide active power and reactive power for the $10 \mathrm{kV}$-ac grid. As the core device of SST system, high frequency

a Corresponding author: redblood_hit@126.com

(C) 2016. The authors - Published by Atlantis Press 
isolation (HFI) DAB converter are attracting more and more attentions for high power density, reduced weight, and low noise without compromising efficiency, cost, and reliability [4-5].

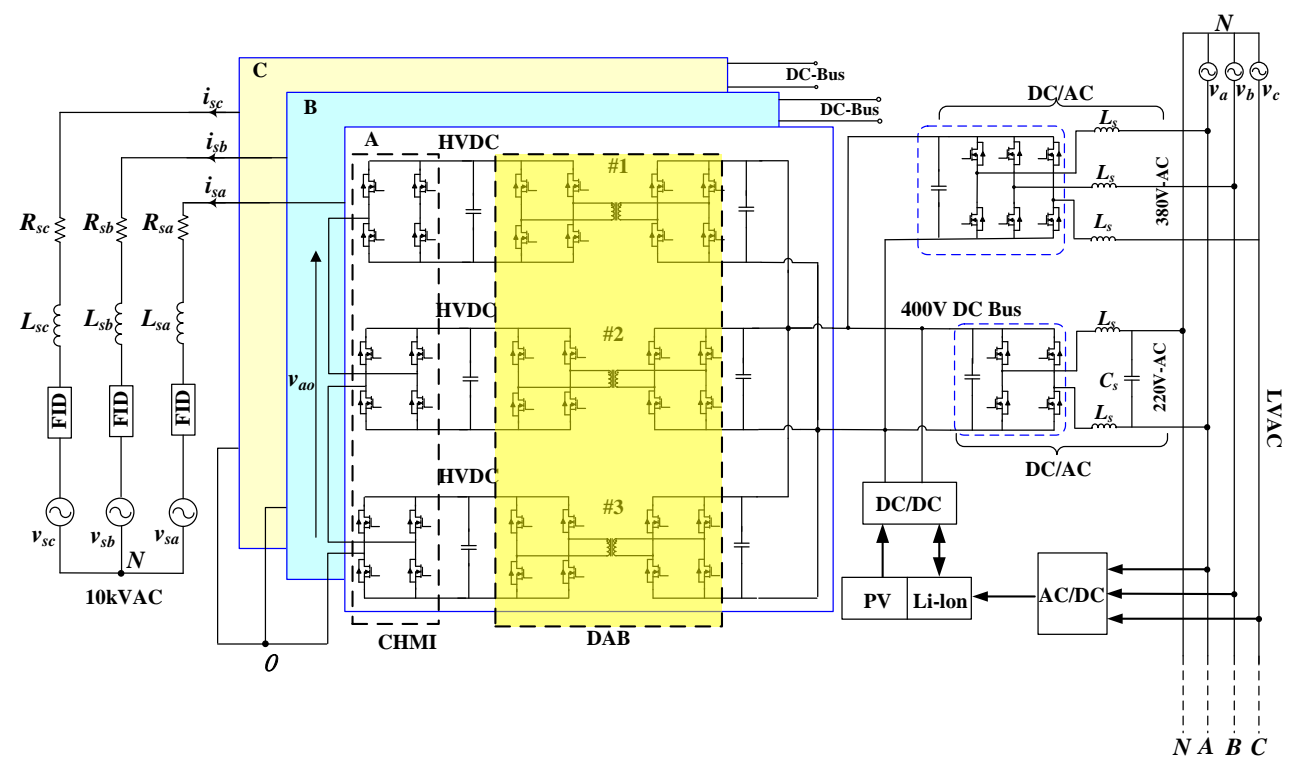

Figure 1. Topology of the proposed three- phase SST.

\section{Dual-active-bridge topology analyses}

Generally, under the same rated voltage and current of switches conditions, the transmission power of converter is proportional to the switches number. Such as the power capacity of four switches is double that of dual-switch, but half that of eight switches, in this case, the dual active bridge is the biggest power capacity. Meanwhile, the filter of DAB is smaller with the same output power than others. In addition, the DAB has the advantages of ease of realizing soft-switching, bidirectional power transfer capability, and modular and symmetric structure, etc. Thus, DAB has been served as the core circuit for the solid state transformer system [6-8].

Fig. 2 shows the topology of DAB, which composes of two H-bridge, two dc link capacitors, an auxiliary inductor, and an HFI transformer. The HFI transformer provides the required galvanic isolation and voltage matching between two voltage levels. The inductor serves as the instantaneous energy storage device.

The transferred power of DAB by phase-shift can be expressed as:

$$
P_{\text {out }}=\frac{n\left(\mathrm{~V}_{d c H} / 2\right)\left(\mathrm{V}_{d c L} / 2\right)(\pi-|\theta|) \theta}{2 \pi^{2} f L}
$$

Where, $L$ is the HF transformer leakage inductance, $V_{\mathrm{dcH}}$ is the high voltage dc-link, $V_{\mathrm{dcL}}$ is the $400 \mathrm{~V}$ dc-link and is the phase-shift angle. In addition, the output current can be given by :

$$
I_{\text {out }}=\frac{P_{\text {out }}}{V_{d c H}}=\frac{n V_{d c L}(\pi-|\theta|) \theta}{8 \pi^{2} f L}
$$




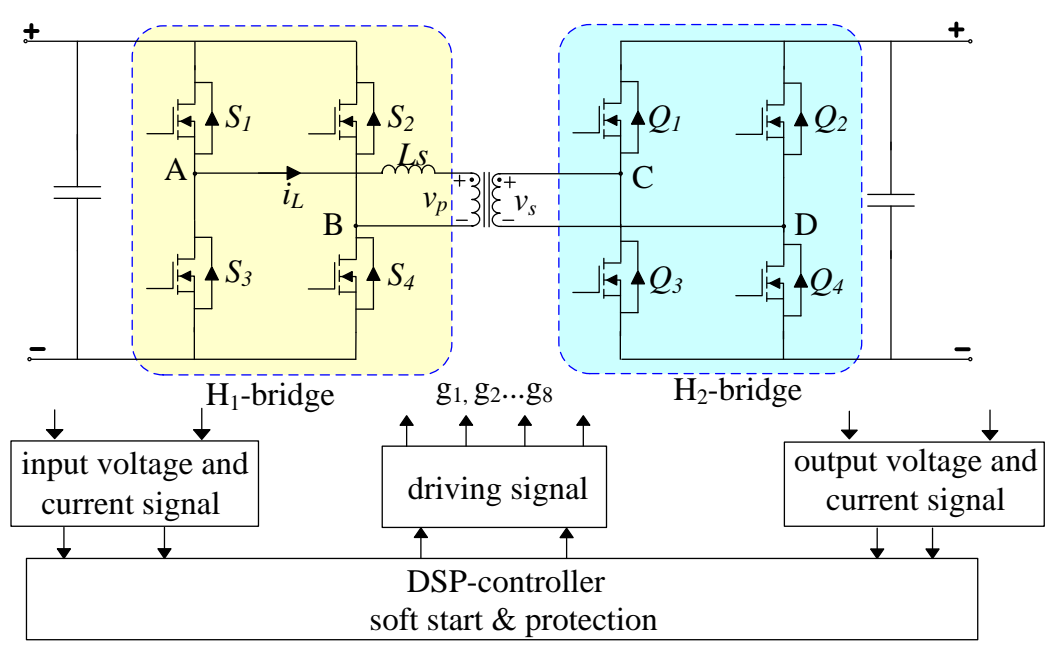

Figure 2. DAB topology and control method.

Based on above, the timing diagram of DAB with single phase-shift control is shown in Fig. 3. The phase-shift angle is adjusted between primary and secondary side, whose range is from 0 to $\pi$. The primary and secondary voltage, $V_{\mathrm{AB}}$ and $V_{\mathrm{CD}}$, can be controlled by the angle [9].

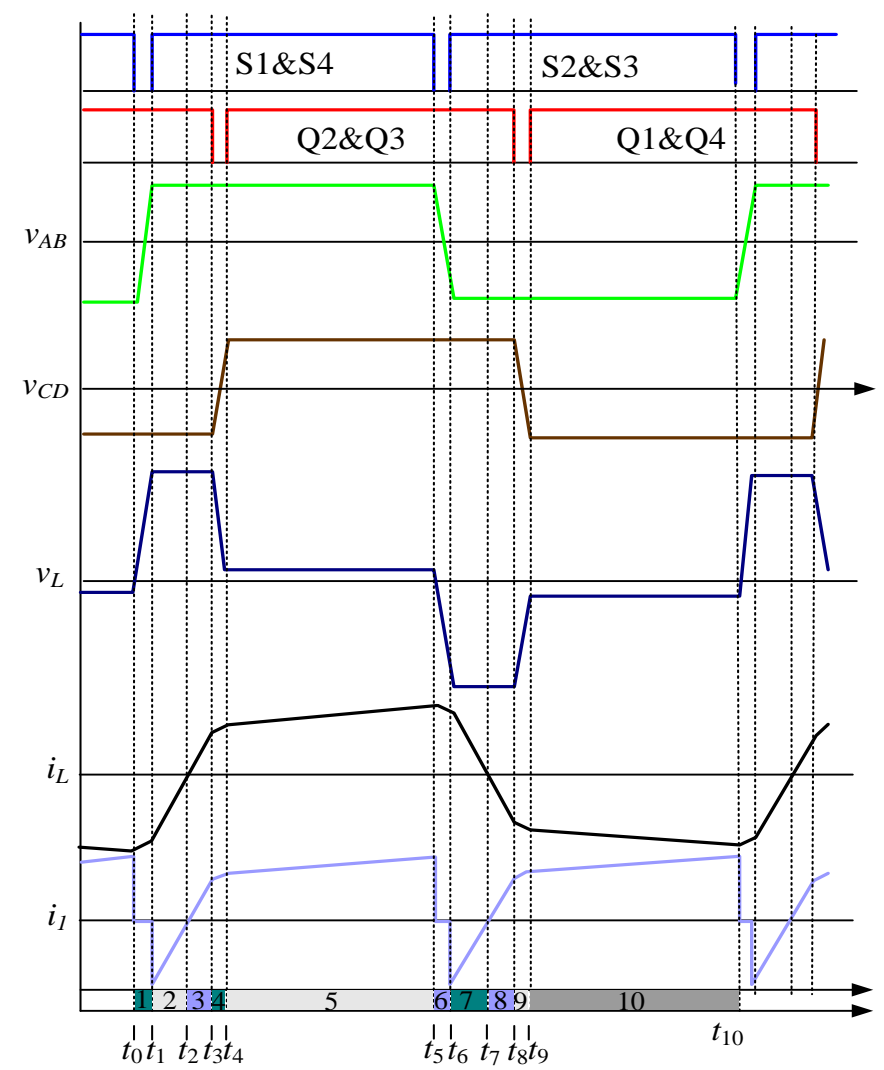

Figure 3. Voltage and current waveforms of DAB control. 


\section{The DAB control scheme optimization based on SST system}

Fig. 4 shows control architecture of the DAB stage with two different methods. The DAB stage steps up the $400 \mathrm{~V}$ dc link to $4 \mathrm{kV}$ dc output which is fed to CHMI stage. The DAB stage consists of 9 DAB modules, if the independent control is used (as shown in Fig. 4a), which will include 9 control loops, resulting in increasing pressure to the software operation and affect the reliability of the system. In contrast, in the proposed control circuit only one DAB converter is utilized as the master PI (proportional-Integral) voltage controller, which executes all control and modulation calculations and sends the resultant converter driving command signals to other DAB slave controllers via high speed fiber-optic (as shown in Fig. 4b).

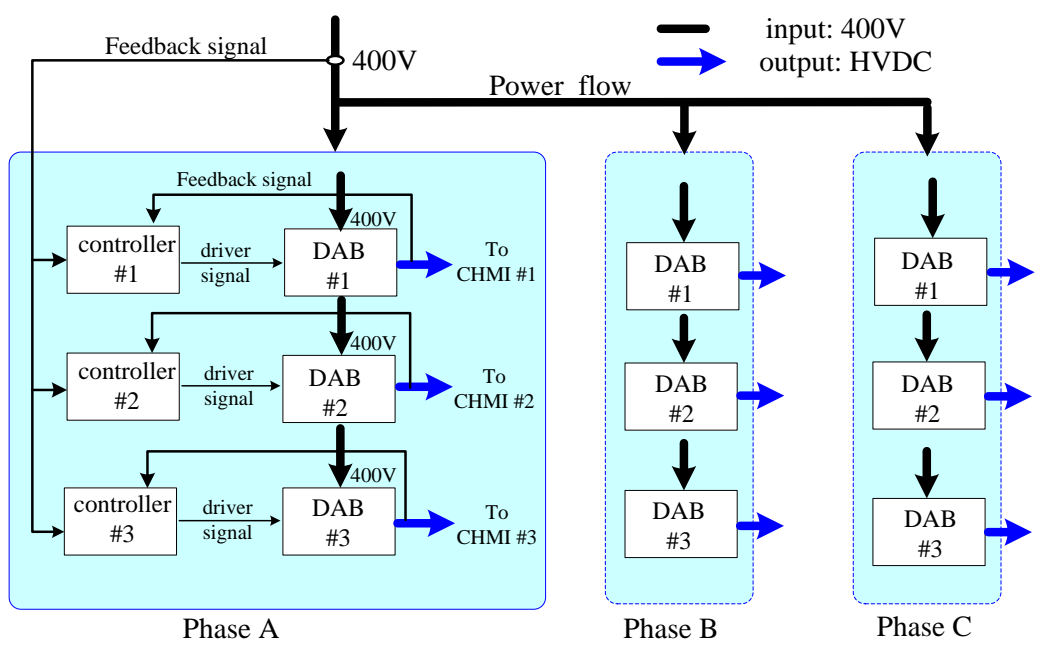

(a) Independent controller

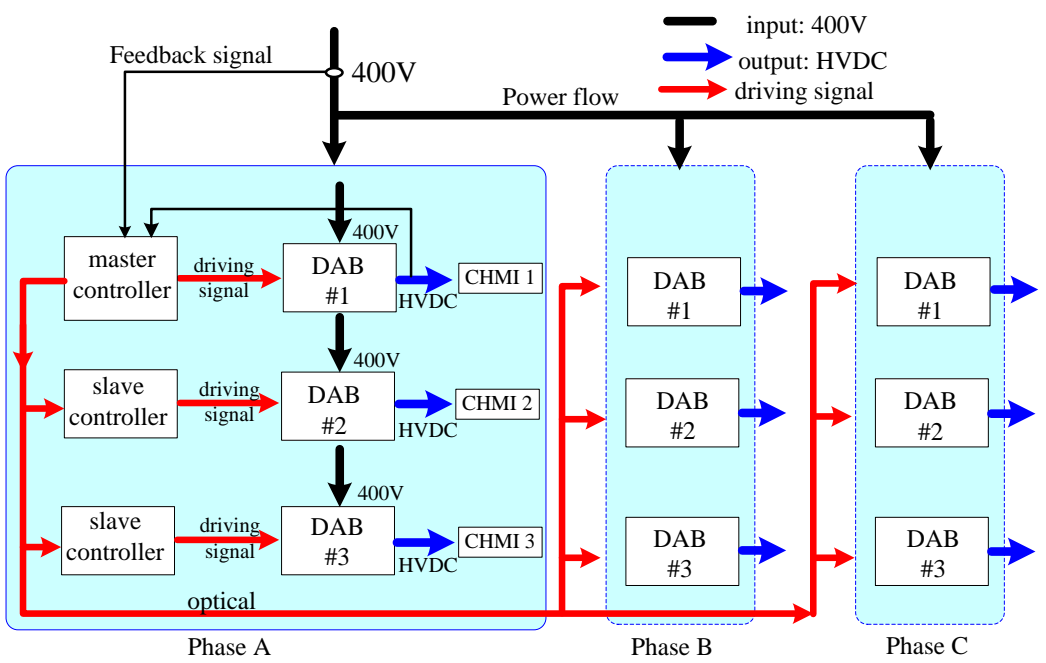

(b) Master-salve controller

Figure 4. The architecture of the DAB stage with two different control strategy.

Typically, 8 DAB slave controllers only manage devices switching and protection. Also to calculate the power transferred by each module and implement the protection of over-voltage and over-current, the voltage and current of input-side and output-side are all sensed. Since 9 circuit structures are completely identical, the output voltage amplitude of each DAB converter should be equal to the reference voltage with the same driving signals assuming that the components (switches 
and transformers) of each DAB stage are ideal. Therefore, the proposed master-slave controller in the DAB stage not only provides the high frequency galvanic isolation, but also simplifies the complex algorithm and improves the dynamic performance.

\section{The simulation and experimental verification}

To verify the proposed DAB controller simulation prototype of Fig. 2 is implemented using Matlab/Simulink. The simulation parameters are listed in Table I.

Table 1. Parameters of the simulation system

\begin{tabular}{|c|c|}
\hline Basic Parameters & Value \\
\hline Input DC Voltage & $400 \mathrm{~V}$ \\
\hline Output DC Voltage & $400 \mathrm{~V}$ \\
\hline Switching Device Frequency & $100 \mathrm{kHz}$ \\
\hline Output power rate & $2.5 \mathrm{~kW}$ \\
\hline Leakage inductance & $5.5 \mathrm{uH}$ \\
\hline DC capacitor (HV/LV) & $5 \mathrm{mF} / 5 \mathrm{mF}$ \\
\hline
\end{tabular}

As shown in Fig. 5, the basic transmission power characterization of DAB with a single phaseshift control is implemented, where $D=/ \pi$ is the phase-shift ratio. Fig. 5 (a) and (b) show the relation curve of transferred power and the phase-shift ratio D. It can be seen that the transmission power of $\mathrm{DAB}$ can be accommodated by $\mathrm{D}$. The transmission current and power increase in the condition of $0 \leq D \leq 0.5$, and the power decreases in the condition of $0.5 \leq D \leq 1$. From Fig. 5(c), the transmission power is symmetric at the median axis $\mathrm{D}=0.5$, and the maximum point of the transmission power is achieved at $\mathrm{D}=0.5$. Furthermore, the power is transferred from high voltage dclink to $400 \mathrm{~V}$ dc-link in the condition of $-1 \leq D \leq 0$.

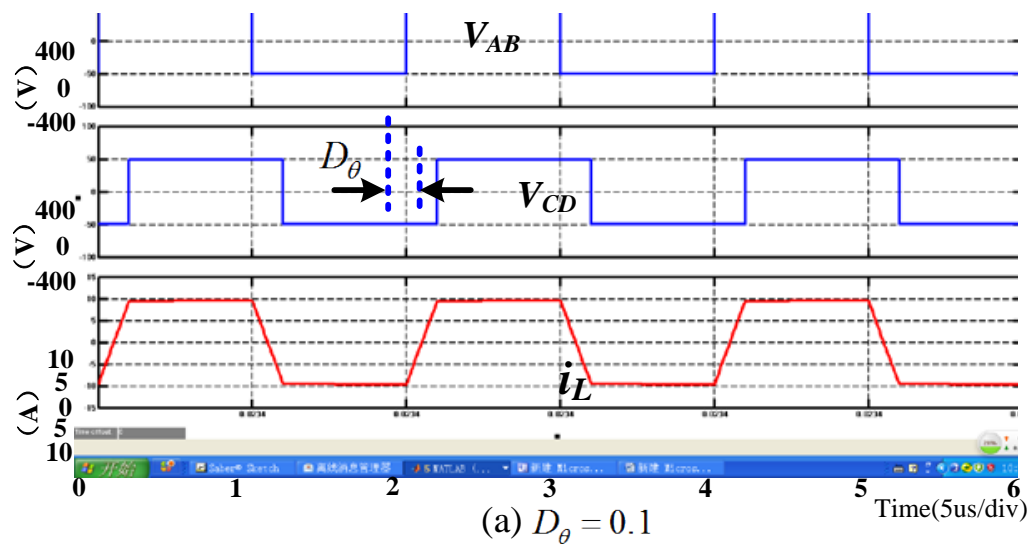



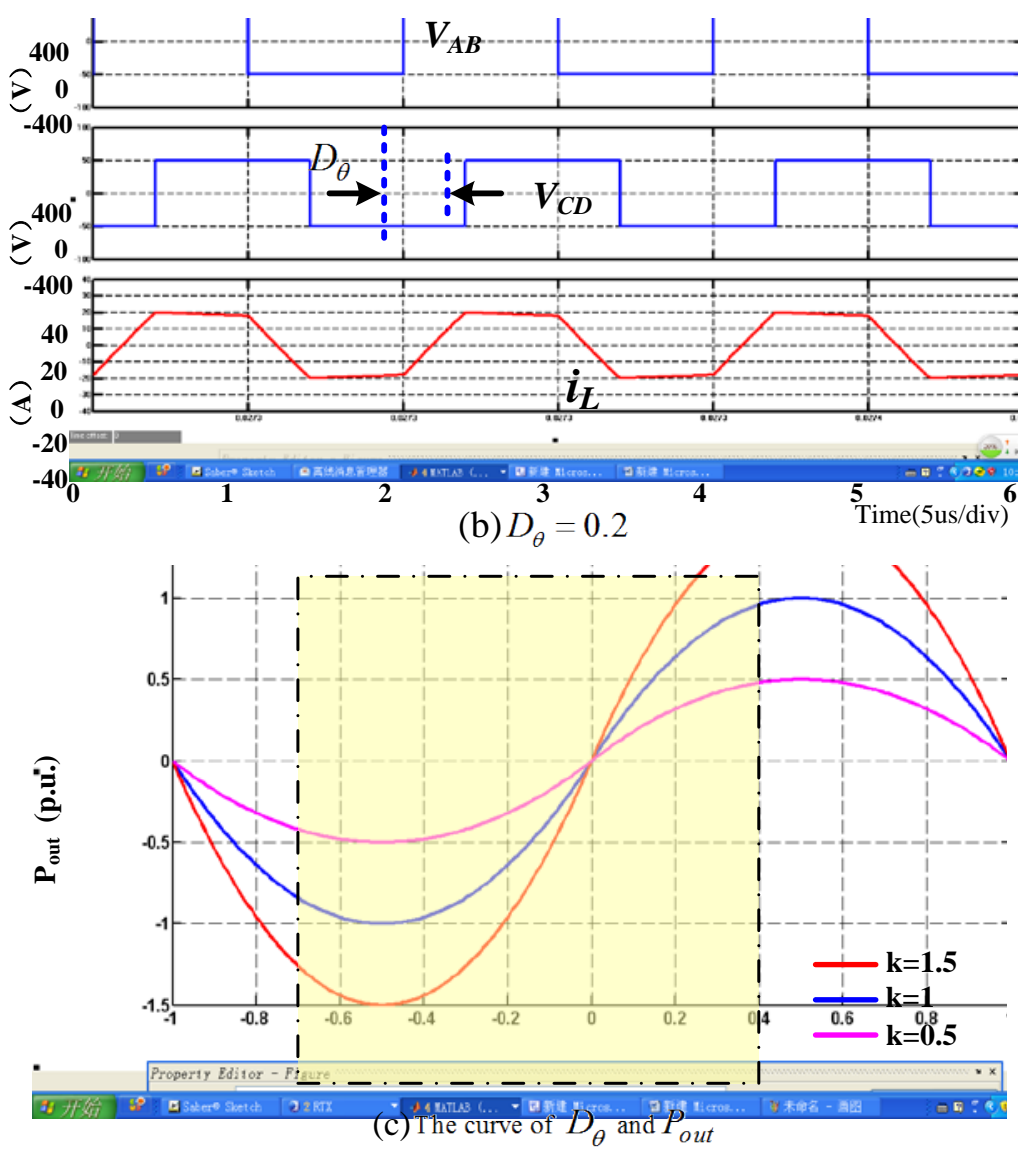

Figure 5. The curve of phase-shift ratio and output power

Fig. 6 shows the experimental waveform of $\mathrm{V}_{\mathrm{GS}}, \mathrm{V}_{\mathrm{DS}}$ and the inductance current $i_{\mathrm{L}}$ under the $V_{\text {in }}=400 \mathrm{~V}$ and $V_{\text {out }}=400 \mathrm{~V}$ operation conditions. From the waveform, the zero-voltage-switching can be achieved [10]. In addition, the 20kVA experimental platform of SST system is shown in Fig. 7.

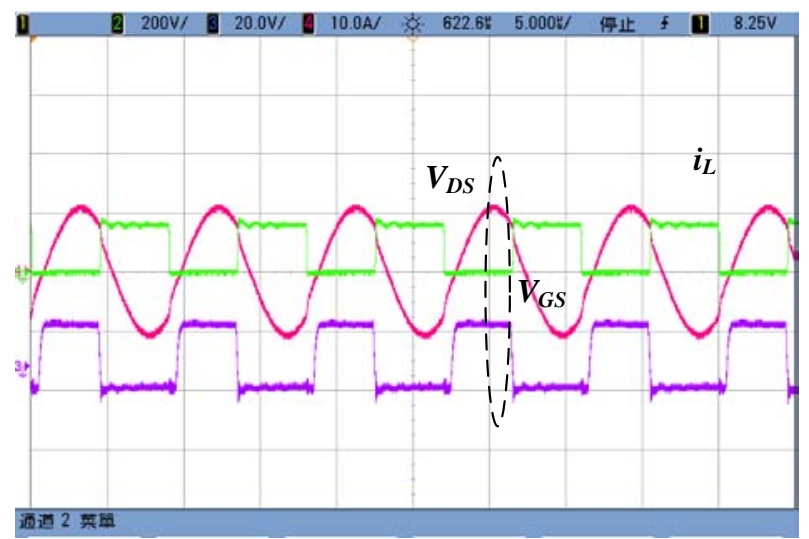

Figure 6. Experimental waveform of $\mathrm{V}_{\mathrm{GS}}, \mathrm{V}_{\mathrm{DS}}$ and $i_{\mathrm{L}}$ 


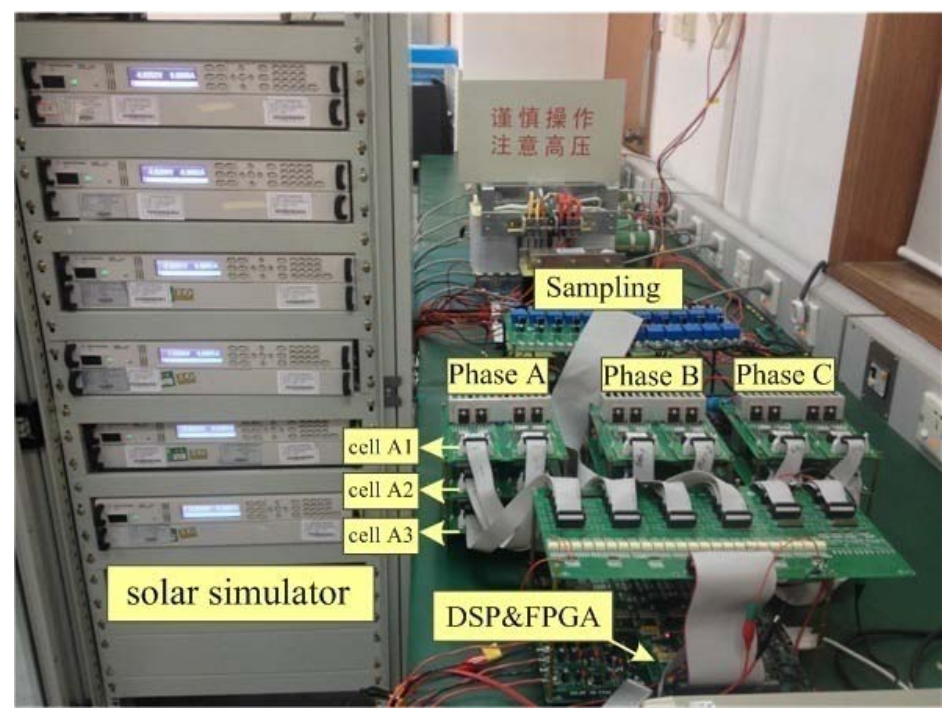

Figure 7. The experimental platform of SST system

\section{Conclusion}

This paper analysis starts from the detailed architecture of solid state transformer, which mainly includes the bidirectional DC/DC converter and the cascaded H-Bridge multilevel inverter. As the core part of the SST system, this paper focused on the master-slave control strategy on the DAB stage. In the proposed control circuit only one $\mathrm{DAB}$ converter is utilized as the master controller, which executes all control and modulation calculations and sends the resultant converter driving command signals to other DAB slave controllers via high speed fiber-optic. The proposed control scheme can simplify the algorithm complexity, improving the operation reliability, expecting to provide theory reference and technology supports for the further SST system development.

\section{Acknowledgements}

This work was supported by the Shenzhen Science and Technology Plan Project (Grant No.: JSGG20140519171924378 and JCYJ20140417172417127).

\section{References}

1. J. Carrasco, L. Franquelo, et al. Power-electronic systems for the grid integration of renewable energy sources: A survey. IEEE Trans. Ind. Electron., 53, 4, 1002-1016(2006).

2. H. Fan and H. Li. High frequency high efficiency bidirectional DC-DC converter module design for 10 kVA solid state transformer," in Proc. IEEE Appl. Power Electron. Conf., 210-215(2010).

3. B. Zhao, Q. Song, et al. Overview of dual active bridge isolated bidirectional dc-dc converter for high frequency link power convertersion system. IEEE Trans. Power Electron., 29, 16, 4091-4106 (2014).

4. J. Lee, Y. Jeong, B. Han. A two stage isolated/bidirecional dc/dc converter with current ripple reduction technique. IEEE Trans. Ind Electron., 59, 3, 644-646(2012).

5. S. Inoue and H. Akagi. A bidirectional dc-dc converter for an energy storage system with galvanic isolation. IEEE Trans. Power Electron., 22, 6, 2299-2306(2007).

6. T. Zhao, G. Wang, et al. Voltage and power balance control for a cascaded h-bridge converter based solid state transformer. IEEE Trans. Power Electron., 28, 10, 1523-1532(2013). 
7. A. Kadavelugu, S. Baek, et aI. High-frequency design considerations of dual active bridge 1200V SiC MOSFET dc-dc converter. 261h Annual IEEE Applied Power Electronics Conference and Exposition. (APEC), 314-320(2011).

8. Y. Xie, J. Sun, et aI. Power flow characterization of a bidirectional galvanically isolated highpower dc/dc converter over a wide operating rang. IEEE Trans. Power Electron., 25, 1, 5466(2010).

9. H. Fan, H. Li. High frequency transformer isolated bidirectional dc-dc converter modules with high efficiency over wide load range for 20kVA solid state transformer. IEEE Trans. Power Electron., 26, 10, 3599-3608(2011).

10. H.Tao, A.Kotsopoulos, et aI. Transformer-coupled multiport ZVS bidirectional dc-dc converter with wide input range. IEEE Trans. Power Electron., 23, 2, 771-781(2008). 